\title{
THE IMPACT OF AN APPLICATION OF TELEREHABILITATION TECHNOLOGY ON CAREGIVER BURDEN
}

\author{
LYN R. TINDALL, ${ }^{1}$ RUTH A. HUEBNER ${ }^{1}$ \\ ${ }^{1}$ Department of Veterans affairs Medical Center, Lexington, Ky
}

\section{ABSTRACT}

The objective of this research was to assess the effects of an application of telerehabilitation reducing time and financial obligation on caregiver burden among eleven caregivers of elderly persons with Parkinson's disease. Clients (care receivers) participated in speech therapy delivered via videophones in their homes; the protocol required 16 treatments delivered four times a week for four weeks. At the conclusion of treatment, caregivers completed a structured interview about the impact of telerehabilitation on time and financial aspects of the burden of care. On average, this speech therapy protocol delivered by videophones saved 48 hours of time, more than 92 hours of work time, and $\$ 1024$ for each caregiver. Savings were significant and previous research demonstrated nearly equal outcomes using the videophone delivery method. Implications for practice and research are discussed.

Keywords: Telerehabilitation, Speech Therapy, Parkinson's Disease

\section{INTRODUCTION}

As the American population ages, a growing number of individuals will serve as caregivers for elders affected by chronic disabling conditions. The vital role of caregivers is well recognized; but their economic and emotional burdens are poorly understood (Caserta, Lund, \& Wright, 1996; Chio, Gauthier, Calvo, Ghiglione, \& Mutani, 2005; Grunfeld, Coyle, Whelan, Clinch, Reyno \& Earle, et al., 2004, 2004; Hauser, \& Kramer, 2004; Hunt, 2003; Novak \& Guest, 1989; Schrag, Hovris, Morley, Quinn \& Jahanshahi, 2006). There is evidence that the presence of caregivers is associated with higher quality of life and reduced morbidity and mortality among care receivers (Schrag et al., 2006). Informal caregivers reduce family and government expenses by delaying or eliminating admission to nursing home facilities among care receivers. Despite these benefits to others, caregivers experience stress associated with the demanding, repetitive, and conflicting challenges of providing care.

Caregiver burden is a term used to describe the physical, emotional, and financial toll of providing care (Parks \& Novielli, 2000). The primary caregiver is most often the spouse, adult child, friend, or distant relative of the care receiver. Caring for an elderly chronically-ill family member is time consuming and associated with persistent stress and impaired psychological functioning of caregivers (Scrag et al., 2006). Caregivers incur substantial financial burden as well. An average family caregiver spends 20.5 hours per week in care giving with $20 \%$ of caregivers having to stop working to care for a family member and $31 \%$ losing all or most of their savings during the final illness of a loved one (Levine, 2000). Findings from a recent survey study conducted by Evercare/National Alliance for Care giving (2007), revealed that half of those caring for someone age 50 years or older spend an average of more than 10 percent of their annual income on care giving expenses, with travel and transportation costs reported as the second most common care giving expense (Evercare National Alliance for Caregiving 2007). One-third of caregivers either make accommodation to their work schedules by using vacation or sick time, or terminate employment due to care giving (Mahoney, Mutschler, Tarlow, \& Liss, 2008). It is essential that health care providers gain a greater understanding of the needs of caregivers, support them, and identify methods to reduce caregiver burden. Identifying ways to reduce or eliminate the time and money associated with transporting patients to and from medical centers and clinics for health services could be a productive and simple step in reducing the burden of giving care.

Although the burden of giving care may be difficult to assess, the measurement of both time and money invested in giving care are frequently used to operationally define components of caregiver burden. For example, transporting a care receiver to a hospital or clinic for 
treatment involves both time costs of waiting during an appointment and financial costs of lost work and travel expenses. Even though time and money costs describe a portion of the obligation of giving care, stressors such as loss of social contacts and personal time vitally contribute to the burden of care. In this study, we focus on reducing caregiver burden by eliminating the time and expense of transporting a care receiver for a speech therapy visit. Specifically, we investigated the effects of videophone delivery of speech therapy services on easing the financial burden of travel and waiting during therapy sessions on caregivers.

In a related study, we examined the comparability of treatment delivered by videophones (Tindall, Huebner, Stemple \& Kleinert, 2008) that substantially reduced the time and costs of travel. We identified equal treatment results on key indicators. This study demonstrated that telerehabilitation technology has the potential of achieving similar therapeutic results when compared to clinicdelivered intervention. We also wanted to explore the effects of videophone-delivered treatment on caregiver burden. In this study we specifically sought to examine financial caregiver burden, but found that the range of published caregiver burden measures were not specific or sensitive enough to measure reduction in financial burden. Although several well developed caregiver burden scales are available (Novak \& Guest, 1989; Zarit, Reever, \& Bach-Peterson, 1980), the global scores obtained from these instruments were judged to be too broad and too subjective for application to this study. Interviews can be helpful in uncovering data difficult to obtain through written responses or observations especially in an emerging field of investigation (Phillips, 1997). We chose to use a structured interview because the data can be quantified similar to a questionnaire with the advantage of ensuring that the responses are understood by the researcher, that the respondent is free to add salient information, and that complete information is collected.

\section{MATERIALS AND METHODS}

Eleven caregivers were recruited for this study. They were identified as primary caregivers by individuals enrolled in a speech therapy program for persons with Parkinson' Disease (PD) who were receiving speech therapy via videophones. To comply with the specific treatment protocol, treatment must be delivered four days a week for four weeks. In the traditional delivery model, caregivers often transport the care receiver to the clinic for treatment. In this study, all therapy sessions were provided in the home using videophones (Tindall et al., 2008) and required only three visits during four weeks to the medical center for baseline and follow-up measures. Caregivers were defined as having primary responsibility for transporting their care receiver to hospital and doctor visits. They completed the structured interview at the conclusion of 16 speech therapy treatments during the follow-up assessment.
The structured caregiver burden interview developed for this study was based on the work of Philips (1997) that provided formulas and strategies for identifying costs and cost avoidance. Philips advises that people can assign a monetary value to changes that cannot be measured objectively. In this structured interview, we asked caregivers to assign a monetary value to the total time they may have spent related to attending and waiting for a speech therapy session for their care receiver. The interview was based on the literature (Zarit et al., 1980; Novak \& Guest, 1989; Caserta et al., 1996) and reviewed by university faculty, Department of Veterans Affairs staff, and piloted on a few caregivers before use in this study. Modifications were made based on feedback.

Specifically, the final structured interview (see Appendix A) included questions about round trip mileage to a clinic for an appointment, the amount of actual time involved driving and waiting for a speech therapy appointment, the amount of actual money used for food, child care, and payment to another individual for driving associated with an appointment. Caregivers assigned a monetary value on the total time spent on a speech therapy appointment. It was expected that this would yield subjective but valuable information about the burden of care not previously analyzed and incorporate the unique circumstances of each caregiver. Finally, caregivers rated their global perceptions of videophone-delivered therapy on the burden of care using a 5-point scale with ' 1 '= Strongly disagree, ' 3 '= Undecided, and ' 5 '= Strongly agree. The instrument was scored using straightforward frequencies and descriptive data about financial value and cost avoidance associated with speech therapy delivered in the home. Financial burden of care for caregivers was defined as time and money for travel and obtained by averaging the amount of time and money actually involved in transporting a care receiver to an outpatient visit.

\section{RESULTS}

Structured caregiver burden interviews were completed by eleven caregivers (seven females, four males). Eight were spouses and three were adult children of care receivers. The average mileage for a round trip visit to the medical center for speech therapy was 93 miles with a range of .5 to 200 miles. The United States Internal Revenue Service (IRS) standard rate of $\$ .48$ per mile was used to calculate the actual cost of mileage. The average amount of time involved for a caregiver to transport a care receiver to and from the medical center and wait until the speech therapy appointment was over was three hours with a range from 1.5 to 5 hours. Seven respondents (64\%) indicated that they had to take between 2.5 to 10 hours time off from work to do this. The mean time taken off from work was 5.8 hours for each visit.

When asked to put a dollar amount on the time involved driving and waiting during one speech therapy visit, 
care receivers responded with a mean of $\$ 94.00$ and a range of $\$ 0$ to $\$ 400.00$. These responses included one participant who replied zero because she felt it was not a burden to provide transportation for her care receiver. Other participants replied with the actual amount of wages involved or the subjective value of their time spent while transporting and waiting for an appointment. The striking variability of responses to this question revealed the range of caregivers affected by caring for a loved one, from retired persons to those actively in the workforce. Further breakdown of responses revealed that seven participants indicated a dollar amount of \$50.00$\$ 80.00$ and two indicated an amount between $\$ 81.00$ and $\$ 100.00$. The median dollar amount reported was $\$ 70.00$. No one reported child care expenses; however, one respondent stated that she paid a driver $\$ 10.00$ to transport her and her care receiver round trip to the speech therapy visit, nine respondents stated that it was necessary to purchase meals with a mean cost of $\$ 20.66$ for two meals and a range of $\$ 15.00$ to $\$ 35.00$ for two meals for each visit.

Using these per visit costs, the total average of hours saved by using videophones for this speech therapy program of 16 treatment sessions was 48 actual hours and 92.8 hours of work time. The average dollar amount saved or avoided by each individual using a videophone for the total speech therapy program was $\$ 1024.00$. This amount includes the IRS standard rate for mileage, average hourly wage rate for each participant, costs of purchasing food, and paying a driver. These values are displayed in Table 1. medical center. Due to the time involved for travel and a

point scale, all caregivers except one rated the reduction of burden as a ' 5 '. They endorsed the reduction of caregiver burden of videophone-delivered speech therapy with a mean score of 4.91 and a range of 4 to 5 .

\section{DISCUSSION}

Caregiver burden is multi-dimensional and affects physical, social, and emotional facets of a caregiver's life. In a progressive disease, such as PD, care-related activities can be expected to increase as the disease progresses. Time demands and financial strain contribute to the perception of caregiver burden. It is incumbent upon health professionals to identify alternatives that might ease burden while achieving positive outcomes. This study explored the effects of therapy delivered by an alternative method of care delivery that eased the time and financial concerns associated with caregiver burden.

The average amount of savings in terms of time and money was significant for participants in this study. The financial burden of transporting a care receiver has been identified as one of the most burdensome aspects of providing care. For individuals who travel long distances to receive care, the cost of gas for their automobile, time off from work for the care receiver, and other cost may prohibit them from accessing care. An added benefit may be the alleviation of apprehension a caregiver may experience when attending to other duties, knowing that a health care professional is watching over the care receiver for a period of time.

Undeniably, these two areas related to caregiver burden were minimized or eliminated with the use of videophones to receive speech therapy. For example, time involved in traveling round trip to a hospital or clinic and waiting for a speech therapy appointment was eliminated in this study. Financial strain, including cost of operating a vehicle, time off from work, purchasing a meal, and paying a driver were also eliminated by using videophones to receive services. When asked if videophone-delivered speech

Table 1. Means and ranges of time and money associated with transporting a care-receiver to speech therapy per visit and the total of 16 visits.

In addition to the financial return, one respondent remarked that she was able to do housework, run errands, and attend to other duties during the therapy session because she felt confident that a professional was with her care receiver during the therapy session. Using the 5 replied with "strongly agree." This study demonstrates that videophone-delivered treatment has the potential to significantly reduce the burden of caring for others and tests a methodology to measure these effects. As other and tested, it is expected that such technology may also ease caregiver burden. therapy eased the burden of care, all but one respondent applications of telerehabilitation technology are pursued 
Caregivers are a critical component in the overall management of elderly individuals with disabling conditions. The burden of care can be overwhelming. Time off from work, providing transportation, and arranging for childcare are aspects of caregiver burden that may be eliminated or diminished by using videophones to deliver healthcare services. Caregivers who participated in this study overwhelmingly agreed that videophone delivered voice therapy greatly decreased the burden associated with transporting a care receiver to a clinic for services and indicated they would use these services again. Future studies should explore the intangible benefits of provide health care via telerehabilitation technology.

Use of telerehabilitation technology should not be recommended solely on the cost savings demonstrated by this study. Clinicians must be responsible to deliver evidenced-based care that is comparable to traditional face-to-face treatment. We must avoid the notion that delivery of health services via telerehabilitation technology is "better than nothing."

Future research should include exploration of the impact of a wide range of telerehabilitation contexts on large diverse sample sizes of caregivers. Further examination of the effect of caregiver responsibilities on the workplace in terms of lost time and wages should also be undertaken. A cost-benefit analysis that paired the cost of missed appointments with caregiver burden may yield an added benefit of using telerehabilitation technology to provide care. Such research is necessary to develop evidenced-based therapies that are cost effective, supportive of caregivers in the workforce, and ease the financial and time-consuming burden of caring for a chronically ill loved one.

\section{ACKNOWLEDGEMENT}

This material is the result of work supported with resources and the use of facilities at the Lexington, Kentucky Department of Veterans Affairs Medical Center.

\section{REFERENCES}

Caserta, M., Lund, D., \& Wright, S.. (1996). Exploring the caregiver burden inventory (CBI): Further evidence for a multidimensional view of burden. International Journal of Aging and Human Development, 43:21-34.

Chio, A, Gauthier, A, Calvo, A, Ghiglione, P, \& Mutani, R. (2005). Caregiver burden and patients' perception of a burden in ALS. Neurology, 64:1780-1782.

Evercare/NAC study reveals that half of caregivers are spending 10 percent of their income to care for an older loved one. (2007, November). Retrieved November 20, 2007, from http://www.medicalnewstoday.com/ articles/89330.php

Grunfeld, E., Coyle, D., Whelan,T., Clinch, J., Reyno, L., Earle, C., (2004). Family caregiver burden: Results of a longitudinal study of breast cancer patients and their principle caregivers. Canadian Medical Association Journal,170:1795-1901.

Hauser, J. \& Kramer, B. (2004). Family caregivers in palliative care. Clinics in Geriatric Medicine, 20: 671688.

Hunt, C. (2003). Concepts in caregiver research.. Journal of Nursing Scholarship, 1: 27-32.

Levine, C. (2000). Always on Call: When Illness Turns Families into Caregivers. New York: United Hospital Fund of New York

Mahoney, D., Mutschler, P., \& Tarlow, B. (2008). Real world implementation lessons and outcomes from the worker interactive networking (WIN) project: Workplace-based online caregiver support and remote monitoring of elders at home. Telemedicine and eHealth, 14(3), 224234.

Novak, M. \& Guest, C. (1989). Application of a multidimensional caregiver burden inventory. The Gerontologist, 29: 798-803.

Parks, S. \& Novielli, K. (2000). A practical guide to caring for caregivers.

American Family Physician, 62: 2613-2623.

Phillips, J. (1997). Return on Investment in Training and Performance Improvement Programs. Houston, Texas: Gulf Publishing Company

Schrag, A, Hovris, A, Morley, D, Quinn, N, \& Jahanshahi, M. (2006). Caregiver-burden in Parkinson's disease is closely associated with psychiatric symptoms, falls, and disability. Parkinsonism and Related Disorders, 12: 35-41.

Tindall, L., Huebner, R. Stemple, J., \& Kleinert, H. (2008). Videophone-Delivered Voice Therapy: A Comparative Analysis of Outcomes to Traditional Delivery for Adults with Parkinson's Disease. Telemedicine and eHealth, 14(10):1070-1077.

Zarit, S., Reever, K., Bach-Peterson, J. (1980). Relatives of the impaired elderly: Correlates of feelings of burden. Gerontologist, 20: 649-655. 


\section{APPENDIX A}

\section{CAREgIVER BURDEN INTERVIEW}

I want you to consider how much time and money you invest to take your care receiver to the VA Medical Center for a speech therapy appointment. Please answer the following questions.

1. How many miles do you travel round trip to the VA for an appointment?

2. How long does it take for you to drive to the VA and back home?

3. How much time do you spend for a speech appointment? Include travel time plus waiting time.

4. Consider how much your time is worth to you. Put a dollar amount on the total time you spent for a speech appointment.

5. Did you buy a meal or snack while at the VA for this appointment?

6. Did you have to take time off from work to bring your care receiver to this appointment? If yes, how much time did you lose from work?

7. Did you have to pay someone for child care while bringing your care receiver to this appointment? If yes, how much?

8. Did you have to pay someone to drive the two of you to this appointment? If yes, how much?

9. Videophone delivered speech therapy eased the burden of taking your care receiver to the VA Medical Center for therapy? Circle one that best describes your answer.

\section{$1=$ Strongly disagree \\ $2=$ Somewhat disagree \\ $3=$ Undecided \\ 4=Somewhat agree \\ $5=$ Strongly agree}


